www.jmscr.igmpublication.org

Index Copernicus Value: 79.54

ISSN (e)-2347-176x ISSN (p) 2455-0450

crossref DOI: https://dx.doi.org/10.18535/jmscr/v7i4.82

Journal Of Medical Science And Clinical Research

IGM Publication

An Official Publication of IGM Publication

\title{
Role of MRI in Evaluation of Avascular Necrosis of Femoral Head: A Prospective Study
}

\author{
Authors \\ Dr Mohammad Zeeshan Saleem ${ }^{1}$, Dr Devidas B Dahiphale ${ }^{2}$, Dr Abhang Apte \\ ${ }^{1}$ Junior Resident, ${ }^{2,3}$ Associate Professor \\ Department of Radiology, MGM Medical College and Hospital Aurangabad431003, Maharashtra \\ *Corresponding Author \\ Dr Mohammed Zeeshan Saleem \\ Junior Resident, Department of Radiology, MGM Medical College and Hospital
}

\begin{abstract}
Introduction: Avascular necrosis (AVN) of femoral head can be defined as cellular death of bone due to loss of blood supply because of any reason. It affects the individuals at a relatively younger age and males are affected more commonly as compared to females. Though the exact pathophysiology is not known, but loss of blood supply causing death of osteocytes is the final component of AVN. Magnetic resonance imaging is reported to be highly sensitive and specific for the diagnosis of $A V N$ and helps in early diagnosis. We conducted this study to analyze role of MRI in evaluation of avascular necrosis of femoral head.

Materials and Methods: This was a prospective study conducted in the department of radiology of a tertiary care medical institute situated in an urban area. 50 patients having avascular necrosis of femoral head were included in this study on the basis of inclusion and exclusion criteria. Institutional ethical committee approved the study and informed consent was obtained from the patients. Demographic details and detailed history was noted in all the cases. MRI of both hips was done in all patients. MRI findings were studied. Statistical analysis was done using SSPE 16.0 software. For statistical purposes $p$ value less than 0.05 was taken as statistically significant.

Results: In this study of 50 patients with avascular necrosis of femoral head there 43 (86\%) males and 7 (14\%) females with M: F ratio of 1:0.16 most of the patients (56\%) were between 20-40 years and the mean age of patients was found to be $38.48 \pm 13.28$ years. AVN of femoral head was present bilaterally in 31 patients (62\%) and unilateral in 19 patients (38\%). The most common risk factor associated with avascular necrosis of femoral head was alcohol consumption (56\%). Most common stage of AVN was found to be Stage II (39.50\%). The most common MRI sign in studied cases was double line sign which was present in 73/81 (90.12\%) femoral heads. Stage II and III AVN was more common in age group of 21-30 years whereas stage IV AVN was more common in age group of 41-50 years. Effusion was present in 57 hip joints. Bone marrow edema was seen in 68 femoral heads out of 81 affected in our study. On the basis of MRI most common compartment to be affected was found to be antero-superior compartment (51.85\%). Finally the analysis of the cases on the basis of Steinberg Staging showed that the out of 81 femoral heads Stage IIIC was the most common stage seen in 25 femoral heads (30.86\%).

Conclusion: Magnetic resonance imaging is highly sensitive and specific imaging technique for the early diagnosis of avascular necrosis of femoral head. Early diagnosis and appropriate intervention in cases with AVN of femoral head is associated with better outcome. This study demonstrates the diagnostic benefits of MRI in early detection of lesion and its properstaging.

Keywords: Avascular necrosis, Femoral Head, Magnetic resonance Imaging, Staging.
\end{abstract}




\section{Introduction}

Avascular necrosis (AVN), also known as osteonecrosis, can be defined as cellular death of bone due to loss of blood supply because of any reason. This loss of blood supply will result in relentless progression of disease resulting in pain, loss of function and collapse. AVN usually involves epiphyseal ends of long bones particularly femur and humeral heads but small bones such as scaphoid and talus are also commonly involved. The disease is seen affecting mainly young men in 3rd or 4th decade of life, is characterized by non-specific symptoms. The clinical features usually depend upon the bone involved but the cardinal symptoms in AVN involving any bone is Pain which progress relentlessly ${ }^{1}$. The pathophysiology of avascular AVN is not entirely understood but various factors causing AVN have a common final effect of loss of blood supply to the affected bone or a part of it. Some of the risk factors for developing AVN are trauma, corticosteroid use, pancreatitis, alcohol, sickle cell disease, infiltrative diseases, radiotherapy, renal transplantation, Gauchers disease, Gout and Caisson disease .Femoral head is one of the common sites for occurrence of AVN. It causes ischemia of femoral head resulting in death of osteocytes eventually causing collapse of femoral head.The disease starts unilaterally and progression to contralateral hip femoral head with bilateral involvement in up to $72 \%$ of the patients $^{2}$.

Non-invasive diagnostic imaging used in detecting avascular necrosis includes plain X-rays, magnetic resonance imaging (MRI), computed tomography (CT) skeletal scintigraphy and $\mathrm{SPECT}^{3}$.Early detection of AVN allows its conservative treatment by core decompression to be effective, with relief of pain and the preservation of normal function of joint. MR imaging plays a very crucial role in detection of early stages of AVN. Nowadays, MRI is the most sensitive diagnostic imaging modality for detection of AVN at an early stage. Magnetic resonance imaging is a noninvasive diagnostic test which is evaluation is more sensitive than plain film radiography; CT scan or skeletal scintigraphy in the diagnosis of $\mathrm{AVN}^{4}$.

Plain X-Ray for the diagnosis of early stages of AVN has an unacceptably low sensitivity and specificity and does not detect early stages (I and II) of AVN. The main role of MRI is diagnosis of avascular necrosis in asymptomatic patients before $\mathrm{x}$-ray changes become apparent ${ }^{5}$. Plain films better depict the subtle morphologic changes of the subchondral cortex. This also applies to the later stages of AVN, when collapse and secondary degenerative disease take place. Distension of the capsular recess by joint effusion has been well correlated with the X-rays stages of AVN of the femoral head. While bone marrow edema detected on MRI is considered a marker of progression to collapse of femoral head ${ }^{6}$. Early detection of AVN of femoral head is crucial since it will allow early decompression and help preserve the joint. In patients who present with signs and symptoms suggestive of AVN and have normal radiographs MRI is the investigation of choice ${ }^{7}$.

We conducted this prospective study to analyze role of MRI in evaluation of avascular necrosis of femoral head.

\section{Materials and Methods}

This was a prospective observational study conducted in the department of Radio-diagnosis of a tertiary care medical college situated in an urban area. The institutional ethical committee duly approved the study and informed consent was obtained from all the patients included in this study. Study conducted as per ICH good clinical practice guidelines. All the patients who were clinically suspected to be having AVN of femoral head and were referred to our department for imaging were included in this study on the basis of a predefined inclusion and exclusion criteria.

Demographic details of all the patients such as age, gender, weight and body mass index was noted. A detailed history with a special emphasis on risk factors for AVN was asked. History of smoking or alcohol consumption was asked. 
History of trauma or steroid intake or history of undergoing radiotherapy was also specifically asked. Possibility of contraindication to MRI was ruled out by specifically asking the history of factors such as cardiac pacemaker, cochlear implants or possibility of pregnancy claustrophobia. Relevant clinical notes and lab parameters were reviewed in all patients undergoing MRI for AVN. Modified Ficat and Arlet, Sterling and Mitchells classifications was determined in all the cases. MRI findings such as presence of bone marrow edema, joint effusion, loss of contour, reduced joint space and presence of double line sign was looked for in all the cases. The statistical analysis was done using statistical software SSPE 16.0 version. Microsoft office was used for preparing charts and graphs.

\section{MRI technique}

The MRI Examination procedure was explained to patients/guardian and informed written consent was obtained. Detailed history of the present complains, past history, habits and ferromagnetic Implants (contraindication) was obtained. Patients were scanned on 1.5 Tesla Philips Achieva MRI Machine using pelvis coil. Multiplanar imaging with appropriate MRI sequences was done in all the cases. The sequences selected were T1 coronal TE $(9 \mathrm{~ms})$, TR (600-650ms) slice thickness $(3 \mathrm{~mm})$, T2 coronal TE $(100 \mathrm{~ms})$, TR (7000-7500 ms) slice thickness $(3 \mathrm{~mm})$, STIR coronal TE (60 ms), TR (5000-6000ms) slice thickness (3-4 mm), T1 Axial TE (9 ms), TR (600-650ms) slice thickness ( $3 \mathrm{~mm})$, T2 Axial TE (100 ms), TR (4500-5000 ms) slice thickness $(3 \mathrm{~mm})$, STIR axial TE (60 ms), TR (5000$6000 \mathrm{~ms})$ slice thickness (3mm), STIR Sagittal TE (60 ms), TR (4000-5000ms) slice thickness (3mm), T2 sagittal TE (100 ms), TR (5000$6000 \mathrm{~ms})$ slice thickness $(3 \mathrm{~mm})$.

\section{Inclusion criteria}

- Patients giving informed consent to be part of the study.

- Clinically suspected cases of AVN: patients with unilateral or bilateral groin, buttock, thigh pain, deformity or limitation of range of hip movement.

- Traumatic cases clinically suspected to have AVN

\section{Exclusion criteria}

- Patients who refused consent.

- Patients with non-AVN causes of femur, thigh or knee pain.

- Patients with contraindications to MR Imaging: patients with ferromagnetic implants, cardiac pacemakers, claustrophobia etc.

\section{Results}

In this study of 50 patients with avascular necrosis of femoral head there $43(86 \%)$ males and 7 (14\%) females with a M:F ratio of 1:0.16.

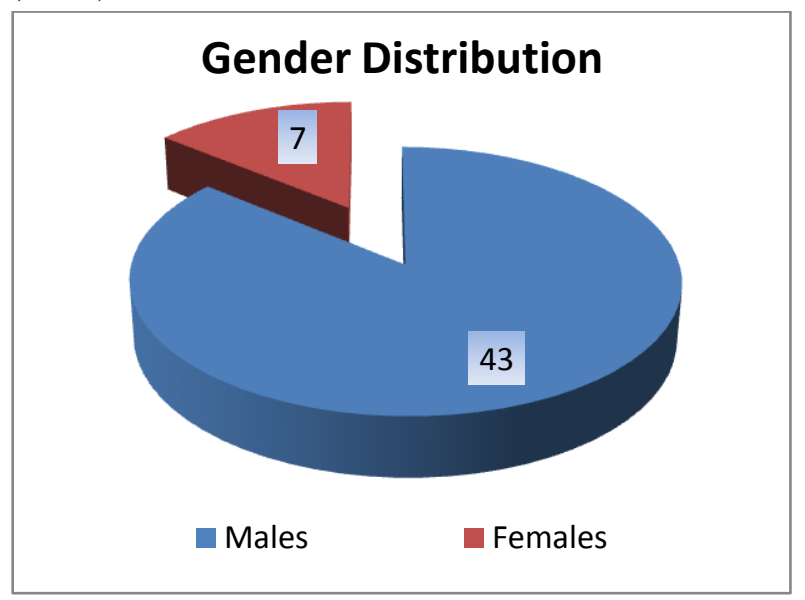

Figure 1: Gender Distribution of the studied cases.

The analysis of the age groups of the studied cases showed that most of the patients (56\%) were between 20-40 years. 13 (26\%) patients belonged to age group of 41-50 years. $3(6 \%)$ patients were below 20 years of age whereas $6(12 \%)$ patients were above age of 50 years. The mean age of patients was found to be $38.48 \pm 13.28$. There was statistically significant difference in the mean age of males and females, and female patients were found to have been affected at a relatively younger age $(\mathrm{P}<0.05)$. 
Table 1: Age group of the studied cases

\begin{tabular}{|lcc|}
\hline Age & No of Patient (n-50) & Percentile \\
\hline$>\mathbf{2 0}$ & 3 & $6 \%$ \\
\hline $\mathbf{2 1}-\mathbf{3 0}$ & 14 & $28 \%$ \\
\hline $\mathbf{3 1}-\mathbf{4 0}$ & 14 & $28 \%$ \\
\hline $\mathbf{4 1}-\mathbf{5 0}$ & 13 & $26 \%$ \\
\hline $\mathbf{5 1}-\mathbf{6 0}$ & 2 & $4 \%$ \\
\hline $\mathbf{6 1}-\mathbf{7 0}$ & 4 & $8 \%$ \\
\hline Total & 50 & $100 \%$ \\
\hline Mean Age $=\mathbf{3 8 . 4 8} \pm \mathbf{1 3 . 2 8}$ & \\
\hline
\end{tabular}

The disease was present bilaterally in 31 patients $(62 \%)$ and unilateral in 19 patients (38\%).

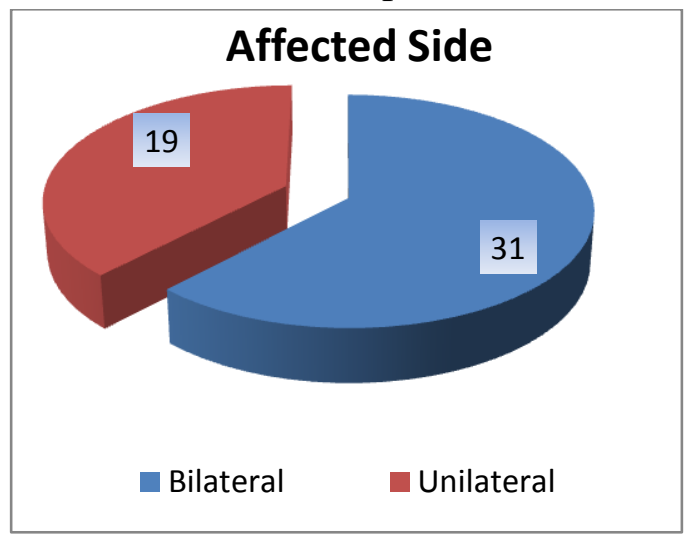

Figure 2: Unilateral vs. Bilateral avascular necrosis in affected cases.

The analysis of the risk factors of the affected cases showed that the most common risk factor associated with avascular necrosis of femoral head was alcohol consumption (56\%) followed by idiopathic AVN (20\%). Steroid use (14\%) and trauma $(16 \%)$ were the other risk factors seen in affected cases.

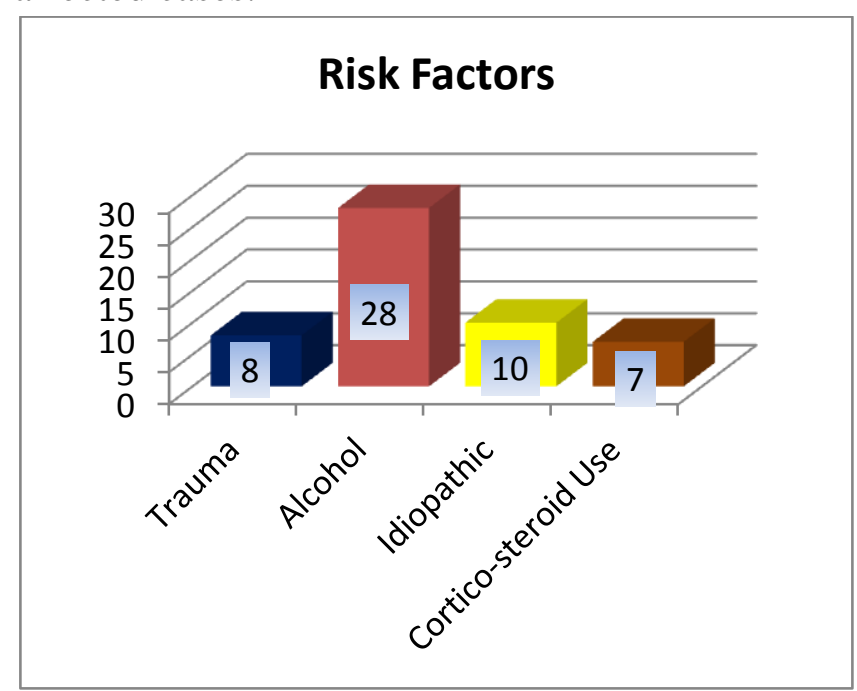

Figure 3: Risk Factors in the studied cases
Stage I AVN was detected in $1(1.23 \%)$ femoral head whereas Stage II AVN was detected in 32 (39.50\%) femoral heads. Stage III and Stage IV AVN was seen in 28 (34.56\%) and 20 (24.69\%) femoral heads.

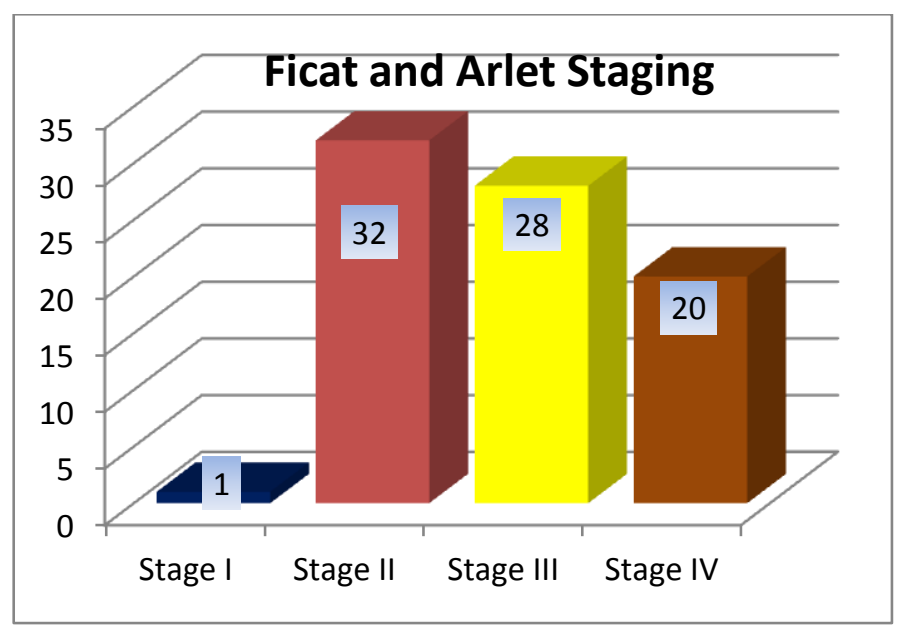

Figure 4: Ficat and Arlet Staging of Avascular necrosis of femoral head.

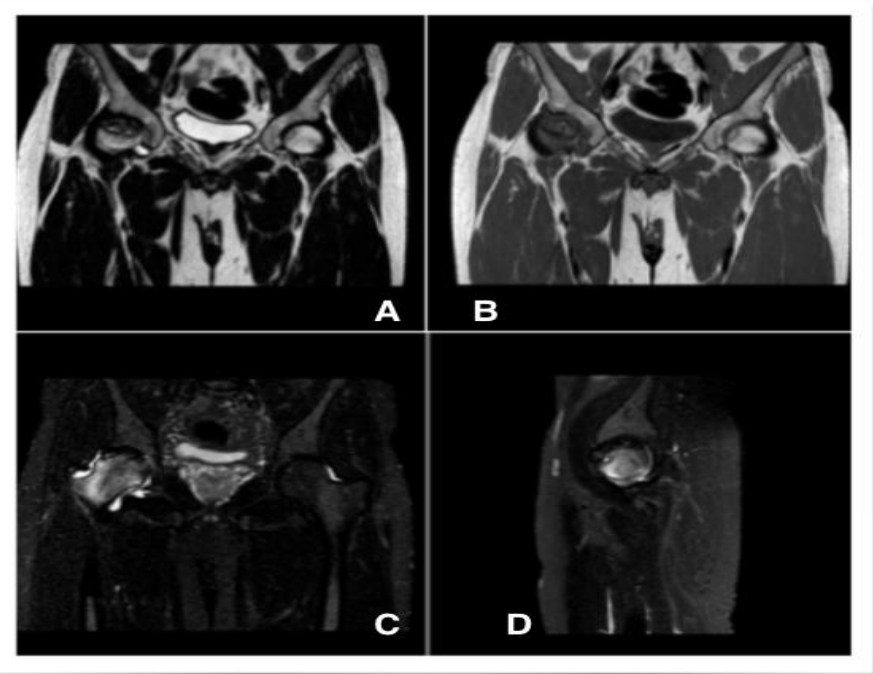

Figure 5: Geographic area of altered signal intensity in right femoral headappearing hypointense on coronal T2W, T1W (A and B) and hyperintense on STIR images (C). Partial collapse with reduced joint space is noted on right side (D). Features s/o stage IV AVN on right side and stage I AVN on left side.

The analysis of MRI findings in the studied cases showed that the most common MRI sign in studied cases was double line sign which was present in 73/81 (90.12\%) femoral heads. The other common MRI findings were Bone marrow 
edema $(83.95 \%)$ followed by joint effusion (70.37\%) and contour loss (59.26\%). Reduced joint space was seen in 20 (24.69\%) femoral heads.

Table 2: MRI findings in the studied cases

\begin{tabular}{|l|c|}
\hline MRI Findings & No of Femoral Heads \\
\hline Double line sign & $\mathbf{7 3}$ \\
\hline Bone Marrow Edema & $\mathbf{6 8}$ \\
\hline Joint Effusion & $\mathbf{5 7}$ \\
\hline Reduced Joint Space & $\mathbf{2 0}$ \\
\hline \hline Contour Loss & $\mathbf{4 8}$ \\
\hline
\end{tabular}

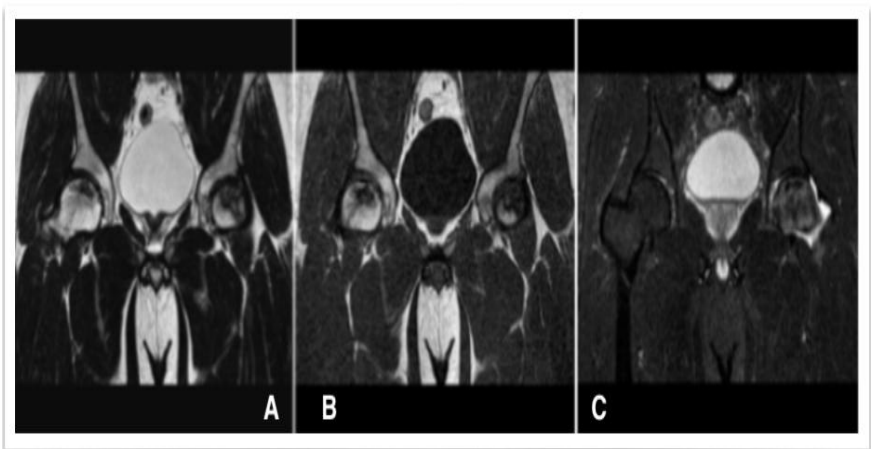

Figure 6: Coronal $\mathrm{T} 2 \mathrm{~W}$ and $\mathrm{T} 1 \mathrm{~W}$ images (A and B) showing areas of altered signal intensity in bilateral femoral heads. Surrounding hypointense rim with inner hyper intense line is seen on coronal T2W images (C) s/o double line sign.

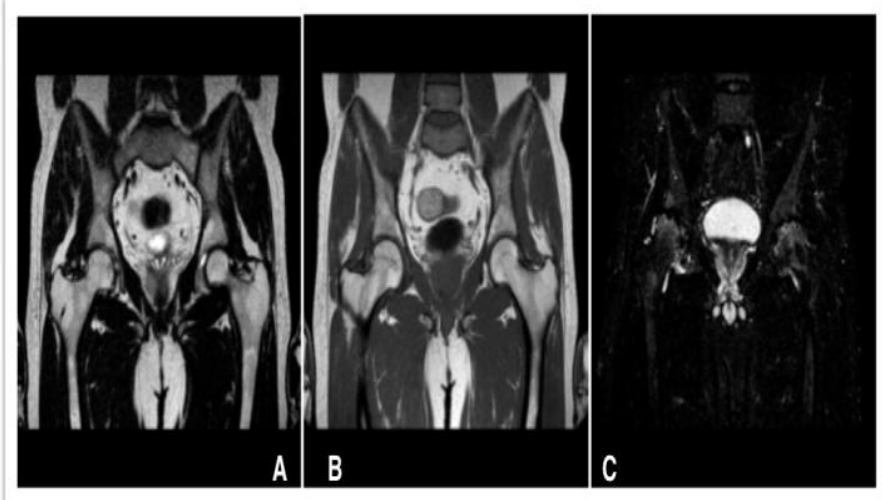

Figure 7: $\mathrm{T} 1 \mathrm{~W}$ and $\mathrm{T} 2 \mathrm{~W}$ coronal images of femoral head appear normal with no signal intensity (A and B). Hyperintense signal intensity on STIR images (C) in right femoral head suggestive of edema. Findings are s/o early changes of avascular necrosis of femoral heads.

The analysis of age group of the patients and its correlation with stage of AVN showed that 9/20 femoral heads with Stage IV belonged to age group of 41-50 years where as 10/28 femoral heads with stage III AVN belonged to age group of 21-30 years. 9/32 patients with stage II AVN belonged to age group of 21-30 years. There was only 1 femoral head with AVN stage I which was seen in a patient of 36 years of age.

Table 3: Association between Age-group\& staging of AVN

\begin{tabular}{|l|c|c|c|c|}
\hline \multirow{2}{*}{ Age } & \multicolumn{4}{c|}{ Stages( Ficat and Arlet) } \\
\cline { 2 - 5 } & I & II & III & IV \\
\hline$>\mathbf{2 0}$ & 0 & 2 & 2 & $\mathbf{0}$ \\
\hline $\mathbf{2 1}-\mathbf{3 0}$ & 0 & 9 & 10 & $\mathbf{4}$ \\
\hline $\mathbf{3 1 - 4 0}$ & 1 & 8 & 6 & $\mathbf{6}$ \\
\hline $\mathbf{4 1 - 5 0}$ & 0 & 8 & 6 & $\mathbf{9}$ \\
\hline $\mathbf{5 1}-\mathbf{6 0}$ & 0 & 2 & 2 & $\mathbf{0}$ \\
\hline $\mathbf{6 1}-\mathbf{7 0}$ & 0 & 3 & 2 & $\mathbf{1}$ \\
\hline \hline Total & $\mathbf{1}$ & $\mathbf{3 2}$ & $\mathbf{2 8}$ & $\mathbf{2 0}$ \\
\hline
\end{tabular}

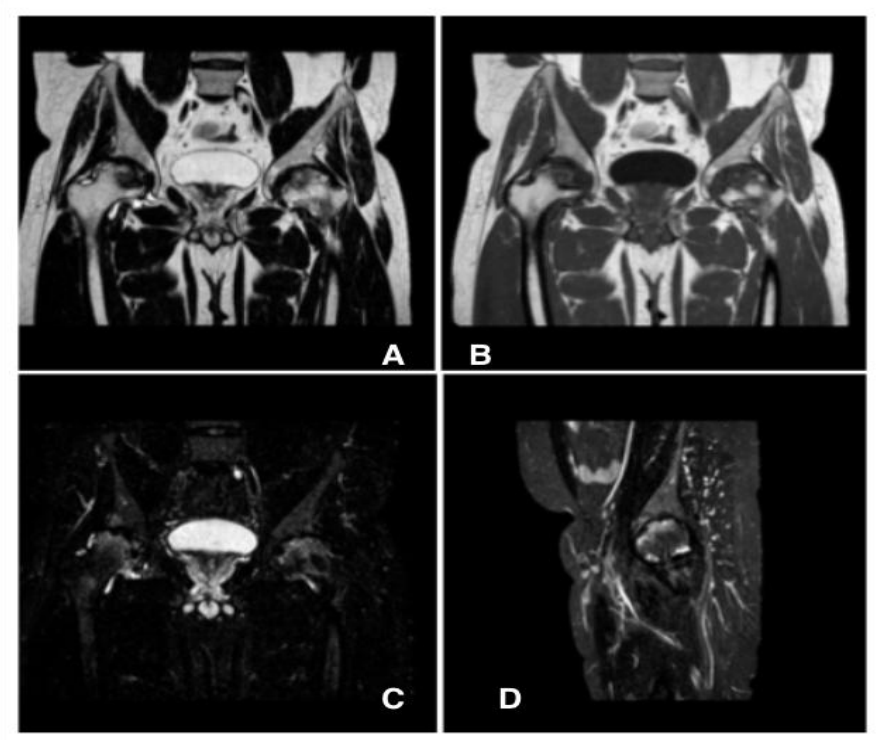

Figure 8: Geographic area of altered signal intensity in superior and anterior quadrant show subchondral sclerosis appearing hypo intense on T2W and T1W images (A and B) STIR and T2W hyperintense signal in bilateral femoral heads s/o Bone marrow edema. (C and D) Finding suggestive of bilateral femoral heads (StageIV FICAT and ARLET Staging).

Out of 81 femoral heads joint effusion was present in 57 hip joints. The incidence of joint effusion was highest in stage III AVN (89.28\%) followed by stage IV AVN (85\%). 15/32 joints (46.87\%) with stage II AVN had effusion whereas effusion was absent in 1 patient with stage I AVN. 
Table 4: Association between Joint effusion \& staging

\begin{tabular}{|l|c|c|c|}
\hline Stage & $\begin{array}{c}\text { Joint } \\
\text { Effusion }\end{array}$ & $\begin{array}{c}\text { No. Of Femoral } \\
\text { Heads }\end{array}$ & Percentage \\
\hline I & 0 & 1 & $\mathbf{0}$ \\
\hline II & 15 & 32 & $\mathbf{4 6 . 8 7 \%}$ \\
\hline III & 25 & 28 & $\mathbf{8 9 . 2 8 \%}$ \\
\hline IV & 17 & 20 & $\mathbf{8 5 \%}$ \\
\hline Total & 57 & 81 & $\mathbf{7 0 . 3 7 \%}$ \\
\hline
\end{tabular}

Bone marrow edema was seen in 68 femoral heads out of 81 affected in our study. In stage II disease the edema was seen in 21 out 32 Femoral Heads $(65.63 \%)$.In stage III disease, the edema extending to the neck region was seen in 26 out of 28 Femoral Heads (92.86\%). All the Femoral Heads affected (100\%) with Stage IV disease showed bone marrow edema in neck and sub trochanteric region in some.

Table 5: Association between Bone marrow edema \& staging

\begin{tabular}{|l|c|c|c|}
\hline Stages & $\begin{array}{c}\text { Bone Marrow } \\
\text { edema }\end{array}$ & $\begin{array}{c}\text { No. Of } \\
\text { femoral heads }\end{array}$ & Percentage \\
\hline I & 1 & 1 & $100 \%$ \\
\hline II & 21 & 32 & $65.63 \%$ \\
\hline III & 26 & 28 & $92.86 \%$ \\
\hline IV & 20 & 20 & $100 \%$ \\
\hline Total & 68 & 81 & $83.95 \%$ \\
\hline
\end{tabular}
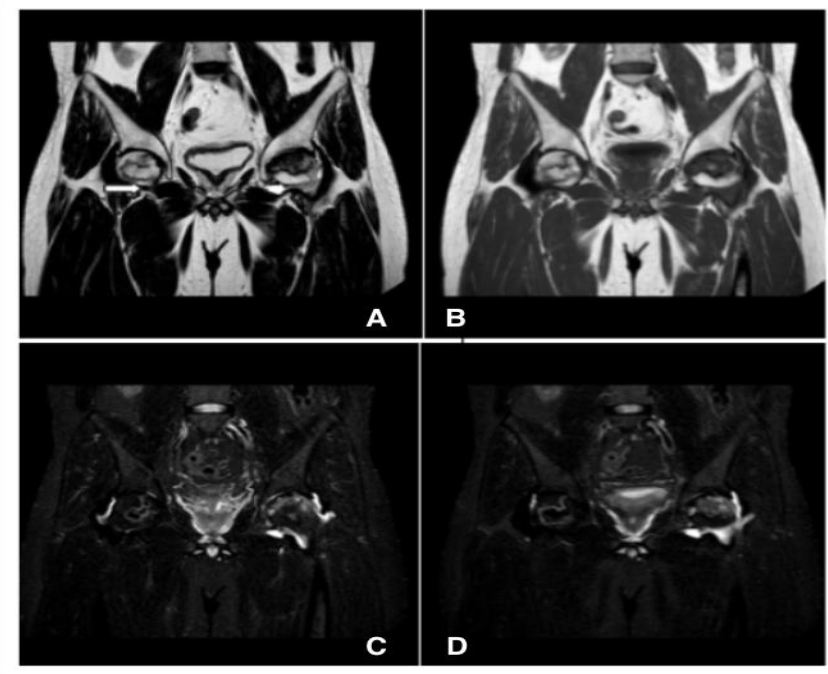

Figure 9: Surrounding hypointense rim with inner hyper intense line is seen on coronal T2W images s/o double line sign (A and B) STIR and T2 FSE (C AND D) hyper-intense signal is noted in left femoral head and neck region s/o bone marrow edema.
The analysis of association of joint effusion with bone marrow edema showed that there was $83 \%$ association of joint effusion with bone marrow edema in Avascular Necrosis of Femoral Heads.

Table 6: Association between Joint effusion \& Bone marrow edema

\begin{tabular}{|l|c|c|c|c|}
\hline \multirow{2}{*}{$\begin{array}{l}\text { Bone Marrow } \\
\text { Edema }\end{array}$} & \multicolumn{2}{|c|}{ Joint Effusion } & & \\
\cline { 2 - 5 } & Present & Absent & Total & Percentage \\
\hline \hline Present & $\mathbf{5 7}$ & $\mathbf{1 1}$ & $\mathbf{6 8}$ & $\mathbf{8 3 . 8 2 \%}$ \\
\hline
\end{tabular}

The analysis of MRI findings and involvement of quadrants showed that out of 81 femoral heads 42 had AVN in antero-superior compartment (51.85\%) followed by anteromedial compartment which was affected in 25 femoral heads (30.86\%). Involvement of complete femoral head was seen in 13 femoral heads $(16.05 \%)$ whereas anterolateral compartment was affected in 1 (1.23\%) patient.

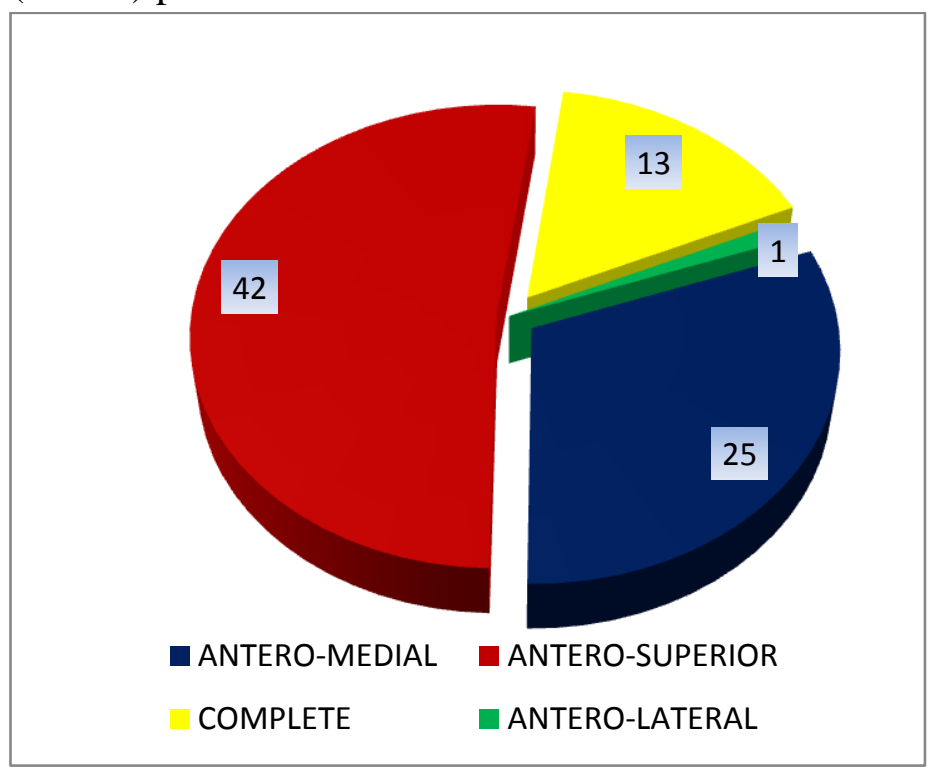

Figure 10: Distribution according to location of lesion in femoral heads.

Mitchells Classification on the basis of signal intensity showed that Type B $(40.74 \%)$ followed by type $\mathrm{D}$ signals (37.04\%) were the most common type of signal intensity lesions seen in our study. Class $\mathrm{C}$ and class A signals were seen in $13(16.05 \%)$ and $5(6.17 \%)$ femoral heads respectively. 
Table 7: Distribution according to signal intensity with lesion of femoral head. (Mitchells Classification)

\begin{tabular}{|l|c|c|}
\hline \multirow{2}{*}{ Class } & \multicolumn{2}{|c|}{ Femoral Heads Involved } \\
\cline { 2 - 3 } & No Of Patients & Percentage \\
\hline A & 5 & $6.17 \%$ \\
\hline B & 33 & $40.74 \%$ \\
\hline C & 13 & $16.05 \%$ \\
\hline D & 30 & $37.04 \%$ \\
\hline TOTAL & 81 & $100.00 \%$ \\
\hline
\end{tabular}
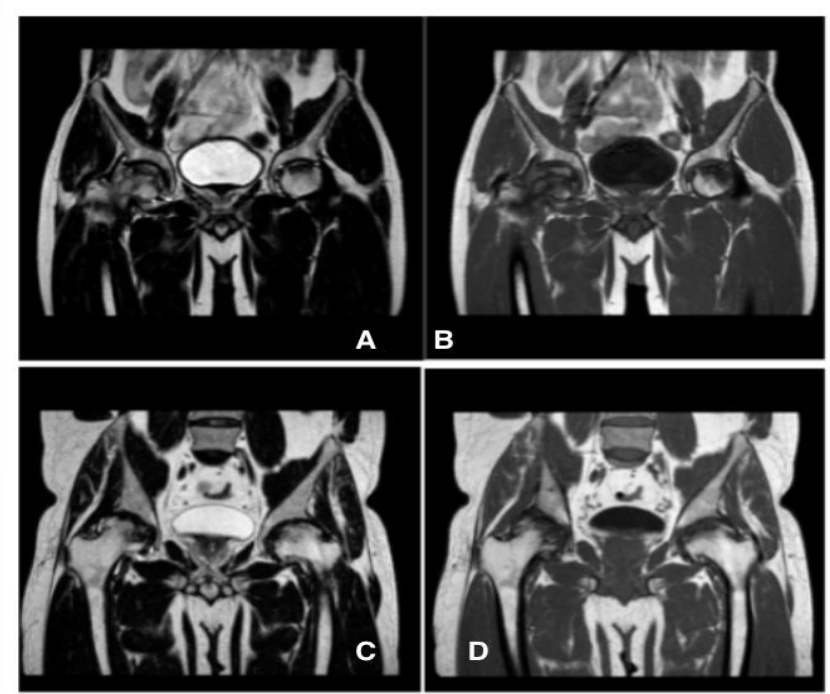

Figure 10: Coronal images (A \& B) shows hyperintense signal on $\mathrm{T} 2 \mathrm{~W}$ and $\mathrm{T} 1$ within the necrotic foci suggestive of Class B (Blood like).Coronal images $(\mathrm{C}$ and $\mathrm{D})$ shows hyper-intense signal on $\mathrm{T} 2 \mathrm{~W}$ images and hypo intense signal on $\mathrm{T} 1 \mathrm{~W}$ images within the necrotic foci suggestive of Class C (Fluid like).

Finally the analysis of the cases on the basis of Steinberg Staging showed that the out of 81 femoral heads Stage IIIC was the most common stage seen in 25 femoral heads $(30.86 \%)$ followed by stage IIC $(23.46 \%)$. Stage VI and IIB were seen in $20.99 \%$ and $12.35 \%$ femoral heads respectively.

Table 8: Steinberg staging of the affected femoral heads

\begin{tabular}{|l|c|c|}
\hline $\begin{array}{l}\text { Steinberg } \\
\text { staging }\end{array}$ & $\begin{array}{c}\text { Affected } \\
\text { Femoral heads }\end{array}$ & Percentage \\
\hline I A & 1 & $1.23 \%$ \\
\hline II A & 3 & $3.70 \%$ \\
\hline II B & 10 & $12.35 \%$ \\
\hline II C & 19 & $23.46 \%$ \\
\hline III A & 0 & 0 \\
\hline III B & 3 & $3.70 \%$ \\
\hline
\end{tabular}

\begin{tabular}{|l|c|c|}
\hline III C & 25 & $30.86 \%$ \\
\hline IV A & 0 & $0 \%$ \\
\hline IV B & 0 & $0 \%$ \\
\hline IV C & 1 & $1 \%$ \\
\hline V A & 0 & $0 \%$ \\
\hline V B & 0 & $0 \%$ \\
\hline V C & 2 & $2.26 \%$ \\
\hline VI & 17 & $20.99 \%$ \\
\hline Total & $\mathbf{8 1}$ & $\mathbf{1 0 0 \%}$ \\
\hline
\end{tabular}

\section{Discussion}

Out of 50 Patients included in our study, 43 (86\%) patients were male and $7(14 \%)$ were female. There is significant higher prevalence of occurrence of AVN in male than female population. Harsha Vardan et al conducted epidemiological survey of Femoral Head AVN in the North Indian Population; they showed male predominance of the disease with male to female ratio of $5: 1^{8}$. Similarly De Wei Zhoa et al in their study showed that the prevalence of AVN was significantly higher in males than in females 9 .

In present study, avascular necrosis was found in the age between 15 to 70 years. The most common age group was found between 21-40 years. The mean age of presentation in male was 38 years and in female 23 years. Harsha Vardan et al in their study had mean age of 34.71 years (range 14-70 years) and $70.28 \%$ patients were between 20 to 40 years ${ }^{8}$.

In this study, alcohol was found to be the most common cause with occurrence of AVN and was seen in 28 patients. Maximum patients i.e. 23 patients out of 28 were found to be in the age group 31 - 40 years with regular drinking habits. In our study history of trauma was seen in 8 patients in whom 2 patients are showing fracture of neck of femur. In 10 patients no specific cause was seen. The use of cortico-steroid was noted in 7 patients. This finding is consistent with the study conducted by Jacobs et al who concluded alcohol as the most common risk factor in $39 \%$ of his cases ${ }^{10}$. Diana Kamal et al in their study found smoking as the most common risk factor found $36.9 \%$ of cases studied ${ }^{11}$.

We found that, in patients with Stage II disease, most of the femoral heads were in $\mathrm{C}$ class i.e. in 
16 patients, followed by B class in 14 patients and A class in 3 patients. Out of all patients with Stage III disease most of them have their femoral heads involved > $30 \%$.In 20 patients with Stage IV disease, 12 were associated with advanced degenerative disease and associated osteoarthritis. In our study we found that 9 hips show $<30 \%$ of femoral head, 40 hips show involvement between $30-50 \%$ and 34 hips shows > $50 \%$ involvement. In patients with involvement of femoral head between 30-50\%, we have seen there is mild contour loss and with patients with > $50 \%$ involvement there is partial to complete femoral collapse. All the hips with Stage IV disease ,6 hips shows partial collapse , 2 hips shows acetabular involvement and 12 shows secondary degenerative changes. Nam K. W et al in his study evaluated fate of asymptomatic AVN with emphasis on size of lesion. If the lesion is seen involving $<30 \%$ of femoral head than there is low risk of collapse, if the lesion is seen involving between $30-50 \%$ than there is moderate risk collapse of collapse and if $>50 \%$ involvement is seen than there is high risk of collapse of femoral head $^{12}$.This finding coincide with Betral et al which showed number of hips undergoing collapse is directly proportional to femoral head involvement; larger the area greater is chance of collapse $^{13}$.

The most commonly area involved was anterosuperior and was seen in each stage. It is seen in $53 \%$ of the cases. The other common area seen involving was antero-medial comprising of $32 \%$ of all the cases. Helena Gabriel et al showed that MRI finding along the antero-superior aspect of the femur are specific ${ }^{14}$. T. Nishi et al in his study showed lesion size and location is prognostic indicator of collapse and large necrotic lesions have likelihood of antero-superior aspect of femoral head ${ }^{15}$.

Present study reveals secondary OA changes was typical feature of Stage IV disease. $86 \%$ of cases with Stage IV disease show secondary OA changes. In this study, Class B as most common finding comprising of $41 \%$ of all the affected hips, followed by Class D comprising of $37 \%$. Class B and Class C are usually seen associated with Stage II and Stage III disease. Class D pattern is seen in Stage IV disease. Similar findings were seen in study conducted by Saurabh Goyal et al which concluded that, Class A signal in necrotic core are usually associated with early changes of AVN while those with Class B and C signal within had advanced Stages ${ }^{16}$.

In our study double line sign was the most common finding seen in 73 out of 81 hips affected. It is most common sign seen in Stage II disease occurring in $96 \%$ of cases with Stage II disease and $86.6 \%$ in cases affected with Stage III disease. Beverly G. Coleman showed similar finding and considered pathognomonic for $\mathrm{AVN}^{17}$.

Bone marrow edema was found out to be most common feature with 68 femoral heads out of 81 $(97.34 \%)$ affected femoral heads in our study. As the stage increases there is more number of femoral heads were seen with marrow edema. In femoral heads with stage II the edema was confined to the necrotic focus which was seen in 21 femoral heads. In stage III disease, the edema extending to the neck region was seen in 26 femoral heads. All the femoral heads affected with Grade IV disease showed edema in neck and trochanteric region. Kim et al. reported that bone marrow edema was most often seen in femoral heads with stage III disease and that marrow edema pattern is characteristic of TO be not seen in earlier stages of AVN of the femoral head ${ }^{18}$.

In our study, joint effusion was found out to be in 57 out 81 affected femoral heads. Our results indicate that the prevalence of JE occurred in hips with stage III disease followed by Stage IV disease. Out of 28 heads with Stage III disease, 25 $(89.28 \%)$ joint effusion and out of 32 heads with Stage II disease $21(65.54 \%)$ had joint effusion. 17 out of $20(85 \%)$ affected heads with stage IV disease have joint effusion. This finding is similar to the study by Mitchell et al. which reported that joint effusion is seen in $58 \%$ of hips with AVN in contrast to $5 \%$ of normal hips ${ }^{19}$.Gou Chu Huang et 
al. showed stage III disease as the most common stage to show joint effusion ${ }^{20}$.

We tried to find the association between joint effusion and bone marrow edema in our study. Out 69 femoral heads having bone marrow edema, $57(82 \%)$ heads had associated joint effusion. In patients with no bone marrow edema, 4 out of 10 patients had effusion. There is $83 \%$ association joint effusion with bone marrow edema in Avascular Necrosis in Hip Joint.Gou Chu Huang etal which reported that there is increased likeliness of occurrence of joint effusion in bone marrow edema ${ }^{20}$.

\section{Conclusion}

Avascular Necrosis of femoral head is increasingly being diagnosed as cause of musculoskeletal disability. Its early diagnosis is important since early interventions are associated with better outcome. MRI is modality of choice for confirmation of diagnosis in individuals clinically suspected to be having AVN and has got a high sensitivity and specificity. This study demonstrates the diagnostic benefits of MRI in early detection of lesion and its properstaging.

\section{Conflict Of Interest: None}

\section{References}

1. Hernigou P. Avascular necrosis of head of femur. Indian J Orthop. 2009;43(1):1-2.

2. Moya-Angeler J, Gianakos AL, Villa JC, $\mathrm{Ni}$ A, Lane JM. Current concepts on osteonecrosis of the femoral head. World $\mathbf{J}$ Orthop. 2015;6(8):590-601. Published 2015 Sep 18.

3. Stoica Z, Dumitrescu D, Popescu M, Gheonea I, Gabor M, Bogdan N. Imaging of avascular necrosis of femoral head: familiar methods and newer trends. Curr Health Sci J. 2009;35(1):23-8.

4. Markisz JA, Knowles RJ, Altchek DW, Schneider R, Whalen JP, Cahill PT. Segmental patterns of avascular necrosis of the femoral heads: early detection with
MR imaging. Radiology 1987; 162:717720.

5. Baig SA, Baig MN. Osteonecrosis of the Femoral Head: Etiology, Investigations, and Management. Cureus. 2018;10(8): e3171. Published 2018 Aug 21.

6. Markisz JA, Knowles RJ, Altchek DW, Schneider R, Whalen JP, Cahill PT. Segmental patterns of avascular necrosis of the femoral heads: early detection with MR imaging. Radiology 1987; 162:717720.

7. Iida S, Harada Y, Shimizu K, et al. Correlation between bone marrow edema and collapse of the femoral head in steroid-induced osteonecrosis. AJR 2000;174:735-743

8. Vardhan H, Tripathy SK, Sen RK, Aggarwal S, Goyal T. Epidemiological Profile of Femoral Head Osteonecrosis in the North IndianPopulation. Indian $\mathbf{J}$ Orthop. 2018;52(2):140-146.

9. Zhao DW, Yu M, Hu K, et al. Prevalence of Nontraumatic Osteonecrosis of the Femoral Head and its Associated Risk Factors in the Chinese Population: Results from a Nationally Representative Survey. Chin Med J (Engl). 2015; 128 (21):284350.

10. Jacobs B. Epidemiology of traumatic and nontraumatic osteonecrosis. Clinical Orthopaedics and Related Research. 1978. 130; 51-67.

11. Diana Kamal, RodicaTraistaru, D.O. Alexandru, D.C.Greecu, L.Mogoanta. Epidemiologic Study of Avascular Necrosis of the Femoral Head. Current Health Sciences Journal. Vol.39, No.3, 2013.

12. Nam KW, Kim YL, Yoo JJ, Koo KH, Yoon KS, Kim HJ. Fate of untreated asymptomatic osteonecrosis of the femoral head. J Bone Joint Surg Am, 2008. 90(3): p. $477-84$ 
13. Beltran J, Burk JM, Herman LJ, Clark RN, Zuelzer WA, Freedy MR, Simon S. Avascular necrosis of the femoral head: early MRI detection and radiological correlation. MagnReson Imaging. 1987;5(6):431-42.

14. Helena Gabriel. MD, Steven WFitzgerald. MD, Melissa T Myers, MD, James S Donaldson, MD. Andrew K Poznanskl, MD. MR Imaging of Hip Disorders, Radiographics 1994; /14.763-781.

15. Takashi Nishii Nobuhiko Sugano Kenji Ohzono Takashi Sakai Yoshinobu Sato Hideki Yoshikawa Significance of lesion size and location in the prediction of collapse of osteonecrosis of the femoral head: a new three-dimensional quantification using magnetic resonance imaging $\mathbf{J}$ Orthop Res. 2002 Jan;20(1):130-6.

16. Saurabh goyal , Purvi desai, Devyani ambadekar, Simranjeet singh correlation between ficat-arlet and mitchell's Staging for avascular necrosis of femur head NJMR, volume 6, issue 4, oct - dec 2016 page 301 .

17. Beverly C. Coleman, MD, Herbert Y. Kressel, MD, Murray K. Dalinka, MD, Mark L. Scheibler, MD, D. Lawrence Burk, MD, Eve K. Cohen, MD, FRCPC. Radiographically Negative Avascular Necrosis: Detection with MR Imaging, Radiology 1988; 168:525-528.

18. Kim YM, Oh HC, Kim HJ. The pattern of bone marrow oedema on MRI in osteonecrosis of the femoralhead. J Bone Joint Surg Br 2000;82:837-841

19. DG Mitchell, V Rao, M Dalinka, CE Spritzer, WB Gefter, L Axel, M Steinberg, and HY Kressel MRI of joint fluid in the normal and ischemic hip American Journal of Roentgenology 1986 146:6, 1215-1218.
20. Guo-Shu Huang. Wing P. Chan, Yue-Cune Chang, Cheng• Yen Chang. Cheng-Yu Chen, Joseph S. Yu. MR Imaging of Bone Marrow Edema and Joint Effusion in Patients with Osteonecrosis of the Femoral Head: Relationship to Pain, AIR 2003, 181: 545-549. 